\title{
Multicomponent Reactions: Microwave-assisted Efficient Synthesis of Dihydropyrimidinones (thiones) and Quinazolinones under Green Chemistry Protocol as Probes for Antimicrobial Activities
}

\author{
M. M. H. Bhuiyan*, Hamidunnessa, and M. M. Mahmud \\ Department of Chemistry, University of Chittagong, Chittagong 4331, Bangladesh \\ Received 3 October 2011, accepted in final revised form 5 December 2011
}

\begin{abstract}
Microwave promoted diammonium hydrogen phosphate, $\left(\mathrm{NH}_{4}\right)_{2} \mathrm{HPO}_{4}$, catalyzed threecomponent Biginelli reaction between an aldehyde, a 1,3-dicarbonyl compound and urea or thiourea under solvent-free conditions afforded the corresponding dihydropyrimidinones and quinazolinones in high yields. The synthesized compounds have been screened for their antimicrobial activity against Pseudomonas aeruginosa, Staphylococcus aureus, Vibrio choloriae, Shigella dysenteriae, Salmonella typhi bacteria and Aspargilllus flavus, Saccharomyces cerevisiae and Candida albicans fungi respectively. Some of the synthesized compounds exhibited pronounced antimicrobial activities.
\end{abstract}

Keywords: Multicomponent reactions; Dihydropyrimidinone; Microwave irradiation; Antimicrobial activity.

(C) 2012 JSR Publications. ISSN: 2070-0237 (Print); 2070-0245 (Online). All rights reserved.

doi: $10.3329 /$ jsr.v4i1.8688

J. Sci. Res. 4 (1), 143-153 (2012)

\section{Introduction}

The multicomponent reactions (MCRs) are one of the most important protocols in organic synthesis and medicinal chemistry [1,2]. The diversity, efficiency and rapid access to small and highly functionalized organic molecules makes this approach of central current interest in the construction of combinatorial libraries and optimization in drug discovery process [3-5]. In addition, MCRs are more environmentally benign and atom economic, as they avoid time-consuming and costly purification processes as well as protectiondeprotection steps. They provide a powerful tool for the one-pot synthesis of diverse and complex compounds as well as small and druglike heterocycles [6]. The 3,4dihydropyrimidin-2(1H)-ones (DHPMs) have recently emerged as important target molecules due to their therapeutic and pharmacological properties [7] such as antiviral [8], antimitotic [9], anticarcinogenic [10], antihypertensive [11,12] and noteworthy, as calcium channel modulators [13]. DHPMs were also screened as neuropeptide antagonists [14],

"Corresponding author: mosharef65@yahoo.com 
agents in treating anxiety [15], optic nerve dysfunction [16] and recently as antioxidant agents [17]. The dihydropyrimidine core unit is also found in nature and in potent HIVgp120-CD4 inhibitors [18]. Therefore, the synthesis of this heterocyclic core unit is of much current importance, and quite a number of synthetic procedures based on the modifications of the century-old Biginelli's reaction [19] involving acid-catalyzed three-component condensation of 1,3-dicarbonyl compound, aldehyde, and urea, have been developed during past few years [20-34]. Basically, these methods are all similar, using different Lewis acid catalysts such as $\mathrm{BF}_{3}$ [20], $\mathrm{FeCl}_{3}$ [21], $\mathrm{InCl}_{3}$ [22], $\mathrm{BiCl}_{3}$ [23], $\mathrm{LaCl}_{3}$ [24], $\mathrm{LiClO}_{4}$ [25], Mn-(OAc) 3 [26], CAN [27] in a solvent such as $\mathrm{CH}_{3} \mathrm{CN}, \mathrm{CH}_{2} \mathrm{Cl}_{2}$, or tetrahydrofuran (THF). Recently, a number of procedures under solvent-free conditions using $\mathrm{Yb}(\mathrm{OTf})_{3}$ [28], montmorillonite [29], ionic liquid [30], $\mathrm{MgBr}_{2}$ [31], $\mathrm{NbCl}_{5}$ [32], silica sulfuric acid [33] and 12-molybdophosphoric acid $\mathrm{H}_{3} \mathrm{PMo}_{12} \mathrm{O}_{40}$ [34] as catalysts have also been reported. However, despite their potential utility, many of these methods involve expensive reagents, stoichiometric amounts of catalysts, strongly acidic conditions, long reaction time, unsatisfactory yields, incompatibility with other functional groups, cumbersome product isolation, difficulties in handling (especially on a large scale), and the solvents used are not at all acceptable in the context of green synthesis. Thus, a practical and more efficient alternative using an inexpensive and environmentally friendly reagent is still of interest for one-pot synthesis of dihydropyrimidinones and thiones under mild conditions.

Many substituted quinazolinones have been found to be physiologically active compounds [35-37] such as antibiotics [38,39], and potent non-nucleoside reverse transcriptase inhibitors of the human immunodeficiency virus (HIV-1) [40]. A number of synthetic methods for the preparation of substituted $(3 H)$-quinazolin-4-ones have been described [41-45]. Recently, the synthesis of the monosubstituted quinazolinones in solution and also dry media has been reported [46,47]. Disubstituted 4-(3H)quinazolinones are also synthesized through a multi-step reaction in solution under microwave irradiation [48-51].

Recently, Microwave heating has emerged as a powerful technique to promote a variety of chemical reactions [52]. Microwave reactions under solvent-free conditions are attractive in offering reduced pollution, low cost and offer high yields together with simplicity in processing and handling [53]. The Biginelli reaction is important for the preparation of dihydropyrimidine derivatives and excellent results are found for reactions carried out with microwave enhancement [54].

Therefore, a great need still exists for versatile, simple and environmentally friendly processes whereby DHPMs may be formed under milder, more practical and microwave conditions. Diammonium hydrogen phosphate, $\left(\mathrm{NH}_{4}\right)_{2} \mathrm{HPO}_{4}$, is a very inexpensive, nontoxic and commercially available compound that can be used in the laboratory without special precautions. To the best of our knowledge, there is only one report regarding the application of diammonium hydrogen phosphate in the preparation of organic compounds under thermal condition [55]. Here we report, for the first time, the use of diammonium 
hydrogen phosphate as catalyst in the synthesis of dihydropyrimidinones and quinazolinones under microwave condition.

\section{Experimental}

\subsection{Physical measurements}

Melting points were recorded with electro thermal melting point apparatus and are uncorrected. Thin layer chromatography was performed on Kieselgel $\mathrm{GF}_{254}$ and visualization was accomplished by iodine vapour or UV Flame. The infrared (IR) spectra were recorded by FTIR spectrophotometer (Model-8900, Shimadzu, Japan) using $\mathrm{KBr}$ matrix in the range 4000-200 $\mathrm{cm}^{-1} .{ }^{1} \mathrm{H}-\mathrm{NMR}$ (400 MHz and $500 \mathrm{MHz}$ ) and ${ }^{13} \mathrm{C}-\mathrm{NMR}$ (100 $\mathrm{MHz}$ and $125 \mathrm{MHz}$ ) spectra were recorded on JEOL GS×400, GEOL JNM-AL 400 (400 $\mathrm{MHz})$ and JEOL GS $\times 400$, GEOL JNM-AL $400(100 \mathrm{MHz})$ spectrometer (internal standard tetramethyl silane) in $\mathrm{CDCl}_{3} \mathrm{CD}_{3} \mathrm{OD}$ and $\mathrm{DMSO}-\mathrm{d}_{6}$ as solvent. Chemical shifts were reported in $\delta$ unit (ppm) with reference to TMS as an internal standard and $J$ values are given in Hz._The carbon, hydrogen and nitrogen percentages in synthesized products were analyzed according to the approved method ASTM D-5291 by employing LecoCHNS-932 analyzer. All reactions were carried out in a commercially available LG microwave oven (MB - 3947C) having a maximum power output of $800 \mathrm{~W}$ operating at 2450 MHz.

\subsection{General procedure for the synthesis of dihydropyrimidinones (thiones) and quinazolinones}

A mixture of aromatic aldehyde (1 mmol), 1,3-dicarbonyl compounds (ethyl acetoacetate, acytylacetone, dimedone) (1 mmol), urea/thiourea $(1.5 \mathrm{mmol})$ and diammonium hydrogen phosphate $(0.3 \mathrm{mmol})$ was irradiated under microwave condition at $160-320$ watt for 60 180 sec. After complete conversion of the reaction (TLC; ethyl acetate: $n$-hexane; 1:5, $\mathrm{v} / \mathrm{v}$ ), the mixture was washed with water to remove excess of urea/thiourea and catalyst diammonium hydrogen phosphate and filtered. The solid mass was recrystallized from ethyl acetate and $n$-hexane solvent mixture.

\subsubsection{Spectral data}

5-Ethoxycarbonyl-6-methyl-4-phenyl-3,4-dihydropyrimidin-2(1H)-one (1): White crystals. IR (KBr) $U_{\max }\left(\mathrm{cm}^{-1}\right)$ : 3244.0, 3116.8, 2979.8, 1726.2 ( $\mathrm{C}=\mathrm{O}$, ester), 1703.0 (C=O, ketonic), 1651.0, 1598.9, 1465.8, 1388, 1290.3, 1091.6, 783.0. ${ }^{1} \mathrm{H}-\mathrm{NMR}\left(500 \mathrm{MHz}, \mathrm{CDCl}_{3}\right): \delta 7.53$ (s, 1H, NH), 7.26-7.33 (m, 5H, Ph), 5.50 (s, 1H, NH), 5.40 (s, 1H, CH, H-4), 4.07 (q, 2H, $\mathrm{OCH}_{2}, J=6.85 \mathrm{~Hz}$ ), 2.36 (s, 3H, $\left.\mathrm{CH}_{3}\right), 1.17$ (t, 3H, CH $\left.3, J=6.85 \mathrm{~Hz}\right) .{ }^{13} \mathrm{C}-\mathrm{NMR}(125 \mathrm{MHz}$, $\left.\mathrm{CDCl}_{3}\right) \delta 165.92,153.42,146.59,143.68,128.51,127.75,126.47,101.05,60.01,55.27$, 
18.10, 13.89. Analysis calculated for $\mathrm{C}_{14} \mathrm{H}_{16} \mathrm{~N}_{2} \mathrm{O}_{3}$ (260.30): C, 64.60; H, 6.20; N, 10.76; Found: C, 64.78; H, 6.04; N, 10.97.

5-Ethoxycarbonyl-6-methyl-4-(4-methoxy-phenyl)-3,4-dihydropyrimidin-2(1H)-thione (2): Yellow crystals. IR $(\mathrm{KBr}) v_{\max }\left(\mathrm{cm}^{-1}\right)$ : 3381.0, 3274.9, 3176.5, $1608.6(\mathrm{C}=\mathrm{O}), 1512.1$ (C=C), 1471.6, 1413.7, 1174.6, 1085.9, 729.0. ${ }^{1} \mathrm{H}-\mathrm{NMR}\left(500 \mathrm{MHz}, \mathrm{CDCl}_{3}\right): \delta 7.21$ (d, 2H, Ar-H, $J=8.60 \mathrm{~Hz}$ ), 6.85 (d, 2H, Ar-H, J=8.60 Hz), 5.35 (s, 1H, CH, H-4), 4.10 (q, 2H, $\mathrm{OCH}_{2}, J=6.85 \mathrm{~Hz}$ ), 3.81 (s, 3H, $\mathrm{OCH}_{3}$ ), 2.36 (s, 3H, $\mathrm{CH}_{3}$ ), 1.56 (bs, 2H, 2×NH), 1.18 (t, $3 \mathrm{H}, \mathrm{CH}_{3}, J=6.85 \mathrm{~Hz}$ ). Analysis calculated for $\mathrm{C}_{15} \mathrm{H}_{18} \mathrm{~N}_{2} \mathrm{O}_{3} \mathrm{~S}$ (306.39): C, 58.80; H, 5.92; N, 9.14; S 10.47; Found: C, 59.24; H, 5.32; N, 8.62; S 9.90.

5-Ethoxycarbonyl-6-methyl-4-phenyl-3,4-dihydropyrimidin-2(1H)-thione (3): Yellow crystals. IR $(\mathrm{KBr}) v_{\max }\left(\mathrm{cm}^{-1}\right)$ : 3327.0, 3174.6, 3105.2, 2981.7, $1672.2(\mathrm{C}=\mathrm{O}), 1571.9$ (C=C), 1465.8, 1326.9, 1197.7, 1116.7, 759.9. ${ }^{1} \mathrm{H}-\mathrm{NMR}\left(400 \mathrm{MHz}, \mathrm{CD}_{3} \mathrm{OD}\right): \delta$ 7.31-7.27 (m, 5H, Ph), 5.30 (s, 1H, CH, H-4), 4.07 (q, 2H, $\mathrm{OCH}_{2}, J=7.32 \mathrm{~Hz}$ ), 2.44 (s, 1H, NH), 2.34 (s, 3H, $\mathrm{CH}_{3}$ ), 2.15 (s, 1H, NH), 1.16 (t, 3H, $\left.\mathrm{CH}_{3}, J=7.32 \mathrm{~Hz}\right) .{ }^{13} \mathrm{C}-\mathrm{NMR}(100 \mathrm{MHz}$, $\left.\mathrm{CDCl}_{3}\right): \delta 176.00,166.97,145.65,144.53,129.51,128.82,127.67,102.81,61.05,56.15$, 17.81, 14.51. DEPT - $90\left(100 \mathrm{MHz}, \mathrm{CDCl}_{3}\right): \delta 129.51,128.82,127.66,56.15$. DEPT 135 (100 MHz, $\left.\mathrm{CDCl}_{3}\right): \delta$ 129.51, 128.82, 127.67, $61.05\left(\mathrm{CH}_{2}\right), 56.16,17.82\left(\mathrm{CH}_{3}\right), 14.51$ $\left(\mathrm{CH}_{3}\right)$. Analysis calculated for $\mathrm{C}_{14} \mathrm{H}_{16} \mathrm{~N}_{2} \mathrm{O}_{2} \mathrm{~S}$ (276.36): C, 60.85; H, 5.84; N, 10.14; S, 11.60; Found: C, 60.80; H, 5.90; N, 10.20; S, 11.65.

5-Acetyl-6-methyl-4-phenyl-3,4-dihydropyrimidin-2(1H)-thione (4): Orange crystals. IR $(\mathrm{KBr}) v_{\max }\left(\mathrm{cm}^{-1}\right): 3280.7,3180.4,3116.8,2997.2,1614.3(\mathrm{C}=\mathrm{O}), 1571.9,1456.2(\mathrm{C}=\mathrm{C})$, 1359.7, 1328.9, 1182.3, 773.4. ${ }^{1} \mathrm{H}-\mathrm{NMR}$ (400 MHz, $\mathrm{CDCl}_{3}$ ): $\delta$ 7.38-7.32 (m, 5H, Ph), 5.45 (s, 1H, CH, H-4), 2.37 (s, 3H, $\mathrm{CH}_{3}$ ), 2.16 (s, 3H, $\mathrm{CH}_{3}$ ), 1.57 (brs, $2 \mathrm{H}, 2 \times \mathrm{NH}$ ). ${ }^{13} \mathrm{C}-\mathrm{NMR}$ $\left(100 \mathrm{MHz}, \mathrm{CDCl}_{3}\right): \delta 196.11,173.93,143.00,141.60,128.83,128.22,126.54,110.86$, 55.50, 29.94, 18.63. DEPT - 135 (100 MHz, $\left.\mathrm{CDCl}_{3}\right): \delta 128.83,128.22,126.53,55.51$, 29.94, 18.63. Analysis calculated for $\mathrm{C}_{13} \mathrm{H}_{14} \mathrm{~N}_{2} \mathrm{OS}$ (246.33): C, 63.39; H, 5.73; N, 11.37; $\mathrm{S}$ 13.02; Found: C, 64.40; H, 5.23; N, 11.62, S 13.43.

5-Acetyl-6-methyl-4-(4-nitrophenyl)-3,4-dihydropyrimidin-2(1H)-thione (5): Orange crystals. IR $(\mathrm{KBr}) v_{\max }\left(\mathrm{cm}^{-1}\right): 3392.6,3099.4,2844.8,1708.8(\mathrm{C}=\mathrm{O}), 1600.8(\mathrm{C}=\mathrm{C})$, 1533.3, 1346.2, 1195.9, 813.9. ${ }^{1} \mathrm{H}-\mathrm{NMR}$ (500 MHz, $\mathrm{CDCl}_{3}$ ): $\delta 8.41$ (d, 2H, H-3', H-5', Ar-H, J= 8.55 Hz), 8.09 (d, 2H, H-2', H-6', Ar-H, $J=6.85$ Hz), 4.13 (s, 1H, CH, H-4), 2.05 (s, 3H, $\mathrm{CH}_{3}$ ), 1.56 (s, 3H, $\mathrm{CH}_{3}$ ), 1.25 (brs, $2 \mathrm{H}, 2 \times \mathrm{NH}$ ). Analysis calculated for $\mathrm{C}_{13} \mathrm{H}_{13} \mathrm{~N}_{3} \mathrm{O}_{3} \mathrm{~S}$ (291.33): C, 53.60; H, 4.50; N, 14.42; S 11.01; Found: C, 54.25; H, 4.30; N, 13.52, S 10.76 .

7,7-Dimethyl-4-phenyl-2-thioxo-2,3,4,6,7,8-hexahydro-1H-quinazolin-5-one (6): Orange crystal. IR (KBr) $\cup_{\max }\left(\mathrm{cm}^{-1}\right): 3082.0,3056.7,3024.2,2962.5,1593.1(\mathrm{C}=\mathrm{O}), 1490.9$ (C=C), 1448.4, 1375.2. ${ }^{1} \mathrm{H}-\mathrm{NMR}$ (500 MHz, $\mathrm{CDCl}_{3}$ ): $\delta 7.27$ (m, 2H, H-3', H-5', Ar-H), 7.18 (m, 1H, H-4', Ar-H), 7.10 (d, 2H, H-2', H-6', Ar-H, J = 8.00 Hz), 5.54 (s, 1H, CH, 
H-4), $2.39\left(\mathrm{~m}, 6 \mathrm{H}, 2 \times \mathrm{CH}_{2}, 2 \times \mathrm{NH}\right), 1.24\left(\mathrm{~s}, 3 \mathrm{H}, \mathrm{CH}_{3}\right), 1.10\left(\mathrm{~s}, 3 \mathrm{H}, \mathrm{CH}_{3}\right) .{ }^{13} \mathrm{C}-\mathrm{NMR}(125$ $\left.\mathrm{MHz}, \mathrm{CDCl}_{3}\right): \delta 190.40,189.32,137.97,128.13,126.68,125.75,115.48,46.95,46.33$, 32.64, 31.32, 29.60, 27.31. DEPT - 90 (125 MHz, $\left.\mathrm{CDCl}_{3}\right): \delta 128.13,126.68,127.75$, 32.64. DEPT - $135\left(125 \mathrm{MHz}, \mathrm{CDCl}_{3}\right): \delta 128.13,126.68,127.75,46.95\left(\mathrm{CH}_{2}\right), 46.33$ $\left(\mathrm{CH}_{2}\right)$, 32.64, $29.60\left(\mathrm{CH}_{3}\right), 27.31\left(\mathrm{CH}_{3}\right)$. Analysis calculated for $\mathrm{C}_{16} \mathrm{H}_{18} \mathrm{~N}_{2} \mathrm{OS}$ (286.40): $\mathrm{C}$, 67.10; H, 6.34; N, 9.78; S 11.02; Found: C, 68.20; H, 5.93; N, 9.36, S 11.45.

7,7-Dimethyl-4-(4-nitrophenyl)-2-thioxo-2,3,4,6,7,8-hexahydro-1H-quinazolin-5-one (7): Yellow crystals. IR $(\mathrm{KBr}) v_{\max }\left(\mathrm{cm}^{-1}\right)$ : 3076.3, 2958.6, $1589.2(\mathrm{C}=\mathrm{O}), 1512.1(\mathrm{C}=\mathrm{C})$, 1411.8, 1373.7, 1344.3, 1299.9, 848.6. ${ }^{1} \mathrm{H}-\mathrm{NMR}$ (400 MHz, $\mathrm{CDCl}_{3}$ ): $\delta 8.15$ (d, 2H, H-3', H-5', Ar-H, J = 6.40 Hz), 7.25 (d, 2H, H-2', H-6', Ar-H, J = $7.80 \mathrm{~Hz}$ ), 5.55 (s, 1H, CH, $\mathrm{H}-4), 2.48-2.36\left(\mathrm{~m}, 6 \mathrm{H}, 2 \times \mathrm{CH}_{2}, 2 \times \mathrm{NH}\right), 1.24\left(\mathrm{~s}, 3 \mathrm{H}, \mathrm{CH}_{3}\right), 1.12\left(\mathrm{~s}, 3 \mathrm{H}, \mathrm{CH}_{3}\right) .{ }^{13} \mathrm{C}-\mathrm{NMR}$ $\left(100 \mathrm{MHz}, \mathrm{CDCl}_{3}\right): \delta 190.89,189.51,146.48,145.97,127.57,123.43,114.79,46.86$, 46.28, 33.15, 31.37, 29.45, 27.35. DEPT - 90 (100.40 MHz, $\left.\mathrm{CDCl}_{3}\right): \delta 127.57,123.43$, 33.15. DEPT - $135\left(125 \mathrm{MHz}, \mathrm{CDCl}_{3}\right): \delta 127.57,123.43,46.86\left(\mathrm{CH}_{2}\right), 46.28\left(\mathrm{CH}_{2}\right)$, 33.15, $29.45\left(\mathrm{CH}_{3}\right), 27.35\left(\mathrm{CH}_{3}\right)$. Analysis calculated for $\mathrm{C}_{16} \mathrm{H}_{17} \mathrm{~N}_{3} \mathrm{O}_{3} \mathrm{~S}$ (331.40): C, 57.99; H, 5.17; N, 12.68; S 9.68; Found: C, 58.65; H, 4.80; N, 12.05; S 9.20.

7,7-Dimethyl-4-(3-methoxyphenyl)-2-thioxo-2,3,4,6,7,8-hexahydro-1H-quinazolin-5-one (8): White crystals. IR (KBr) $\cup_{\max }\left(\mathrm{cm}^{-1}\right)$ : 3056.9, 2933.5, $1595.0(\mathrm{C}=\mathrm{O}), 1490.9,1423.4$ $(\mathrm{C}=\mathrm{C}), 1375.2$, 1163.0, 1037.6, 788.8. ${ }^{1} \mathrm{H}-\mathrm{NMR}\left(400 \mathrm{MHz}, \mathrm{CDCl}_{3}\right): \delta 7.18(\mathrm{t}, 1 \mathrm{H}, \mathrm{H}-5$ ', Ar-H, J = 8.24 Hz), 6.69 (dd, 3H, H-2', H-4', H-6', Ar-H, J = 8.24 Hz, 9.6 Hz), 5.52 (s, $1 \mathrm{H}, \mathrm{CH}, \mathrm{H}-4), 3.74$ (s, 3H, $\left.\mathrm{OCH}_{3}\right), 2.39\left(\mathrm{~m}, 6 \mathrm{H}, 2 \times \mathrm{CH}_{2}, 2 \times \mathrm{NH}\right), 1.24$ (s, 3H, $\left.\mathrm{CH}_{3}\right), 1.10$ (s, 3H, $\left.\mathrm{CH}_{3}\right) .{ }^{13} \mathrm{C}-\mathrm{NMR}\left(100 \mathrm{MHz}, \mathrm{CDCl}_{3}\right): \delta 190.28(\mathrm{C}=\mathrm{O}), 189.28(\mathrm{C}=\mathrm{S}), 159.43$, 139.74, 128.97, 119.10, 115.44, 112.84, 110.10, 54.92, 46.93, 46.28, 32.65, 31.25, 29.59, 27.21. DEPT - $90\left(100 \mathrm{MHz}, \mathrm{CDCl}_{3}\right): \delta 129.08,119.20,112.93,111.07,32.74$. DEPT 135 (100 MHz, CDCl $): \delta 128.97,119.10,112.84,110.97,54.92\left(\mathrm{OCH}_{3}\right), 46.93\left(\mathrm{CH}_{2}\right)$, $46.27\left(\mathrm{CH}_{2}\right), 32.65,29.59\left(\mathrm{CH}_{3}\right), 27.21\left(\mathrm{CH}_{3}\right)$. Analysis calculated for $\mathrm{C}_{17} \mathrm{H}_{20} \mathrm{~N}_{2} \mathrm{O}_{2} \mathrm{~S}$ (316.43): C, 64.53; H, 6.37; N, 8.85; S 10.13; Found: C, 65.20; H, 5.92; N, 9.00, S 10.78.

\subsection{Antimicrobial screening}

The synthesized compounds (1-8) were screened for antibacterial activity against five pathogenic organisms: Pseudomonas aeruginosa, Staphylococcus aureus (ATCC 6538), Vibrio choloriae, Shigella dysenteriae (AE 14396) and Salmonella typhi (AE 14612 (Table-1) and antifungal activity against three organisms: Aspargilllus flavus, Saccharomyces cerevisiae and Candida albicans (Table-2). The disc diffusion method [56] and poisoned-food technique [57] were used for antibacterial and antifungal activities respectively.

The tested compounds were dissolved in $N, N$-dimethylformamide (DMF) to get a solution of $1 \mathrm{mg} \mathrm{mL}^{-1}$. The inhibition zones were measured in millimeters at the end of an incubation period of $48 \mathrm{hrs}$ at $(35 \pm 2)^{\circ} \mathrm{C}$. DMF alone showed no inhibition. Nutrient agar 


\section{Multicomponent Reactions}

(NA) and potato dextrose agar (PDA) were used as basal media to test the bacteria and fungi, respectively. Commercial antibacterial Ampicillin and antifungal Nystatin were also tested under similar conditions for comparison.

Table 1. Antibacterial activity of the synthesized compounds.

\begin{tabular}{cccccc}
\hline \multirow{2}{*}{ Comp. No. } & \multicolumn{5}{l}{ Diameter of zone of inhibition in $\mathrm{mm}(100 \mathrm{mg}(\mathrm{dw}) /$ disc $)$} \\
\cline { 2 - 6 } & $\begin{array}{c}\text { Pseudomonas } \\
\text { aeruginosa }\end{array}$ & $\begin{array}{c}\text { Staphylococcus } \\
\text { aureus }\end{array}$ & $\begin{array}{c}\text { Vibrio } \\
\text { choloriae }\end{array}$ & $\begin{array}{l}\text { Shigella } \\
\text { dysenteriae }\end{array}$ & $\begin{array}{l}\text { Salmonella } \\
\text { typhi }\end{array}$ \\
\hline 1 & -- & 10 & 10 & -- & -- \\
2 & 11 & 14 & -- & 11 & 12 \\
3 & 13 & 11 & -- & -- & 14 \\
4 & 9 & -- & 9 & 11 & -- \\
5 & -- & -- & -- & 14 & -- \\
6 & -- & 9 & -- & -- & 11 \\
7 & 12 & 14 & -- & 11 & 13 \\
8 & -- & 12 & 9 & -- & -- \\
Ampicillin & 20 & 12 & 17 & 30 & 24 \\
\hline
\end{tabular}

Table 2. Antifungal activity of the synthesized compounds.

\begin{tabular}{cccc}
\hline & \multicolumn{3}{c}{ \% inhibition of mycelial growth $(100 \mu \mathrm{g}(\mathrm{dw}) / \mathrm{ml} \mathrm{PDA})$} \\
\cline { 2 - 4 } Comp. & $\begin{array}{c}\text { Aspargillus } \\
\text { flavus }\end{array}$ & $\begin{array}{c}\text { Sacchharromyces } \\
\text { cerevisiae }\end{array}$ & $\begin{array}{c}\text { Candida } \\
\text { albicans }\end{array}$ \\
\hline 1 & 70.90 & 62.67 & 19.46 \\
2 & 86.42 & 85.33 & 68.68 \\
3 & 63.14 & 85.33 & 61.97 \\
4 & 61.20 & 93.33 & 23.94 \\
5 & 76.72 & 84.00 & 83.80 \\
6 & 74.78 & 49.33 & 70.51 \\
7 & 53.54 & 81.33 & 9.10 \\
8 & 61.20 & 66.67 & 14.00 \\
Nystatin & 90.00 & 85.00 & 85.00 \\
\hline
\end{tabular}

\section{Results and Discussions}

Salehi and co-workers [55] have reported the synthesis of DHPMs under solvent-free conventional heating conditions at $80^{\circ} \mathrm{C}$. Herein we have successfully carried out the same transformation under microwave irradiation in comparatively shortest duration. A variety of aldehydes were condensed with ethyl acetoacetate/acetylacetone/dimedone and urea (or thiourea) and the products were obtained in good to excellent yields (Scheme 1 and Table 3). 


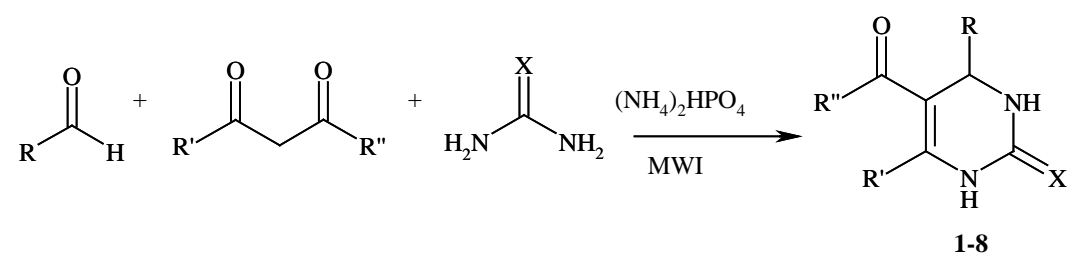

Scheme 1. Synthesis of DHPMs.

Table 3. Synthesis of DHPMs Using $\left(\mathrm{NH}_{4}\right)_{2} \mathrm{HPO}_{4}$ under microwave condition.

\begin{tabular}{|c|c|c|c|c|c|c|c|}
\hline $\begin{array}{l}\text { Comp. } \\
\text { No. }\end{array}$ & $\mathrm{R}$ & $\mathrm{R}^{\prime}$ & $\mathrm{R}^{\prime \prime}$ & $\mathrm{X}$ & $\begin{array}{c}\text { Watt/ } \\
\text { Time(sec) }\end{array}$ & $\begin{array}{c}\text { Yield } \\
(\%) \\
\end{array}$ & $\frac{\text { M.P. }{ }^{\circ} \mathrm{C}}{\text { Found / Reported }}$ \\
\hline 1 & $\mathrm{C}_{6} \mathrm{H}_{5}$ & Me & EtO & $\mathrm{O}$ & $160 / 120$ & 96 & $202-203$ \\
\hline 2 & 4-MeO- $\mathrm{C}_{6} \mathrm{H}_{4}$ & Me & EtO & S & $160 / 90$ & 85 & $\begin{array}{l}203-204[55] \\
150-152 \\
150-151[58]\end{array}$ \\
\hline 3 & $\mathrm{C}_{6} \mathrm{H}_{5}$ & $\mathrm{Me}$ & EtO & $S$ & $160 / 90$ & 95 & $\begin{array}{l}208-210 \\
208-211[55]\end{array}$ \\
\hline 4 & $\mathrm{C}_{6} \mathrm{H}_{5}$ & $\mathrm{Me}$ & $\mathrm{Me}$ & S & $160 / 90$ & 90 & $\begin{array}{l}185-186 \text { (dec) } \\
186 \text { (dec) [55] }\end{array}$ \\
\hline 5 & $4-\mathrm{NO}_{2}-\mathrm{C}_{6} \mathrm{H}_{4}$ & \multicolumn{2}{|c|}{ R'R', } & $S$ & $160 / 120$ & 84 & $213-214$ \\
\hline 6 & $\mathrm{C}_{6} \mathrm{H}_{5}$ & \multicolumn{2}{|c|}{$-\mathrm{CH}_{2} \mathrm{C}\left(\mathrm{CH}_{3}\right)_{2} \mathrm{CH}_{2}-$} & S & $320 / 120$ & 78 & $251-253$ \\
\hline 7 & $4-\mathrm{NO}_{2}-\mathrm{C}_{6} \mathrm{H}_{4}$ & \multicolumn{2}{|c|}{$-\mathrm{CH}_{2} \mathrm{C}\left(\mathrm{CH}_{3}\right)_{2} \mathrm{CH}_{2^{-}}$} & S & $320 / 180$ & 85 & $217-219$ \\
\hline 8 & 3-MeO- $\mathrm{C}_{6} \mathrm{H}_{4}$ & \multicolumn{2}{|c|}{$-\mathrm{CH}_{2} \mathrm{C}\left(\mathrm{CH}_{3}\right)_{2} \mathrm{CH}_{2^{-}}$} & S & $160 / 60$ & 83 & $189-191$ \\
\hline
\end{tabular}

An important feature of this procedure is the survival of variety of functional groups such as nitro and ether under the reaction conditions. Thiourea also reacts under similar reaction conditions to form the corresponding 3,4-dihydropyrimido-2(1H)-thiones in good to excellent yields. The structures of the products were deduced from their IR, ${ }^{1} \mathrm{H}$ NMR, ${ }^{13} \mathrm{C}$ NMR and elemental analyses. For example the ${ }^{1} \mathrm{H}$-NMR spectrum of the compound 7 displayed a deshielded doublet signal resonated at $\delta 8.15(\mathrm{~J}=6.40 \mathrm{~Hz})$ corresponding to two aromatic protons, H-3' and H-5' ortho to the nitro group. Another doublet resonated at $\delta 7.25(J=7.80 \mathrm{~Hz})$ corresponding to two aromatic protons were assigned to $\mathrm{H}-2$ ' and $\mathrm{H}-6$ '. One $\mathrm{CH}(\mathrm{H}-4)$ proton of the quinazolinone ring resonated at $\delta 5.55 \mathrm{ppm}$ was observed as singlet. A multiplet appeared at $\delta$ 2.48-2.36 integrated for six protons corresponding to $2 \times \mathrm{CH}_{2}$ and $2 \times \mathrm{NH}$. The spectrum showed two three-proton singlets at $\delta$ 1.24 and $\delta 1.12$ due to two $\mathrm{CH}_{3}$ protons located at $\mathrm{C}-7$ position. The ${ }^{13} \mathrm{C}$ NMR spectrum of compound 7 showed the presence of thirteen signals attributed to sixteen carbons of corresponding molecular formula $\mathrm{C}_{16} \mathrm{H}_{17} \mathrm{~N}_{3} \mathrm{O}_{3} \mathrm{~S}$. The ${ }^{13} \mathrm{C}$ NMR spectrum showed the existence of a carbonyl group $(\mathrm{C}=\mathrm{O})$ at $\delta 190.89$, a thioxo group $(\mathrm{C}=\mathrm{S})$ at $\delta 189.51$, besides five quaternary carbons resonated at $\delta 146.48$ (C-8a), 145.97 (C-1', C-4'), 114.79 (C-4a) and 31.37 (C-7). Two $\mathrm{CH}_{3}$ carbons observed at $\delta 29.45$ and 27.35. At the same 
time two $\mathrm{CH}_{2}$ groups and C-4 carbons appeared at $\delta 46.86,46.28$ and 33.15. On the other hand, signals resonated at $\delta 127.57$ and 123.43 were ascribed for the four aromatic carbons. The DEPT-90 spectrum of the compound 7 showed the presence of three signals at $\delta 127.57,123.43,33.15$ attributed to five $\mathrm{CH}$ groups in the molecule. The DEPT-135 spectrum of the compound 7 showed the presence of four signals at $\delta 127.57,123.43$ and 31.15 attributed to five $\mathrm{CH}$ groups and two signals resonated at $\delta 29.45$ and 27.35 attributed to two $\mathrm{CH}_{3}$ groups located at C-7 in the molecule. Two carbons of two $\mathrm{CH}_{2}$ groups appeared at $\delta 46.86$ and 46.28 as negative values. The microanalytical data of the compound $\mathbf{7}$ is also in good agreement with the assigned structure. Similarly the peaks in ${ }^{1} \mathrm{H}$-NMR and ${ }^{13} \mathrm{C}$-NMR spectra of the rest compounds were accordance with assigned structures.

Amongst the synthesized compounds screened for the antibacterial activity, compound 2 showed highest activity against $S$. aureus. Some of the compounds showed low antimicrobial activities and some were unable to show inhibition. For the antifungal activity all compounds, except 7, showed excellent results against all fungi. Compound 2, $\mathbf{3}$ and $\mathbf{5}$ revealed highest activity against $S$. cerevisiae, which was also approximately similar to that of the standard antibiotic, Nystatin. The other tested compounds also exhibited good to excellent results against all the fungi.

\section{Conclusion}

In this work, we have developed a new solvent-free strategy for the preparation of 3,4dihydropyrimidin-2-(1H)-ones (and -thiones) as biologically interesting compounds via the condensation of aldehyde with 1,3-dicarbonyl compounds and urea or thiourea. The advantages of this method are high yields, relatively short reaction times, low cost, simple experimental and isolation procedures, and finally, it is in agreement with the green chemistry protocols. The activity data obtained during the study will be certainly useful to go for further research for drug designing and synthesizing new dihydropyrimidinone derivatives.

\section{Acknowledgment}

The authors wish to thank Dr. A. Rahman, Department of Biochemistry and Molecular Biology, University of Chittagong, Bangladesh, for his cooperation in determining the antimicrobial activity of the synthesized compounds. We also wish to thank Dr. M. AlAmin, JSPS Postdoctoral Fellow, Department of Chemistry, Hokkaido University, Japan for recording the spectral data.

\section{References}

1. L. Weber, Drug. Disc. Today 7, 143 (2002). http://dx.doi.org/10.1016/S1359-6446(01)02090-6

2. A. Domling, Curr. Opin. Chem. Biol. 6, 306 (2002). http://dx.doi.org/10.1016/S13675931(02)00328-9 
3. R. E. Dolle and K. H. Nelson, J. Comb. Chem. 1, 235 (1999). http://dx.doi.org/10.1021/cc9900192

4. L. A. Thompson and J. A.Ellman, Chem. Rev. 96, 555 (1996). http://dx.doi.org/10.1021/cr9402081

5. E. M. Gordon, M. A. Gallop, and D. V. Patel, Acc. Chem. Res. 29, 144 (1996). http://dx.doi.org/10.1021/ar950170u

6. B. M. Trost, Angew. Chem. Int. Ed. 34, 259 (1995). http://dx.doi.org/10.1002/anie.199502591

7. C. O. Kappe, Eur. J. Med.Chem. 35, 1043 (2000). http://dx.doi.org/10.1016/S0223$\underline{5234(00) 01189-2}$

8. E. W. Hurst and R. Hull, J. Med. Pharm. Chem. 3, 215 (1961). http://dx.doi.org/10.1021/jm50015a002

9. T. U. Mayer, T. M. Kapoor, S. J. Haggarty, R. W. King, S. I. Schreiber, and T. J. Mitchison, Science 286, 971 (1999). http://dx.doi.org/10.1126/science.286.5441.971

10. T. Kato, Jpn. Kokay Tokkyo Koho 59 190, 974 (1984), (Chem. Abstract 1985, 102,132067).

11. K. S. Atwal, B. N. Swanson, S. E. Unger, D. M. Floyd, S.Moreland, A.Hedberg, and B. C. O’Reilly, J. Med. Chem. 34, 806 (1991). http://dx.doi.org/10.1021/jm00106a048

12. G. C. Rovnyak, K. S. Atwal, A.Hedberg, S. D. Kimball, S.Moreland, J. Z. Gougoutas, B. C. O’Reilley, J.Schwartz, and M. F. Malley, J. Med. Chem. 35, 3254 (1992). http://dx.doi.org/10.1021/jm00095a023

13. C. O. Kape, Molecules 3, 1 (1998). http://dx.doi.org/10.3390/30100001

14. K. S. Atwal, G. C. Rovnyak, J. Schwartz, S. Moreland, A. Hedberg, J. Z. Gougoutas, M. F. Malley, and D. M. Floyd, J. Med. Chem.. 33, 1510 (1990). http://dx.doi.org/10.1021/jm00167a035

15. Z. P. Horovitz (E.R. Squibb, and Sons), Eur Pat Appl., EP 400, 665 (1990), [Chem.Abstr., 115, 64793 (1991)].

16. C. E. Crosson, D. E. Potter, M. A. Ondetti, D. Floyd, and G. Aberg, (Houston Biotechnology, Inc.; Squibb E R, and Sons) PCT Int. Appl. WO 06, 118 (1990), [Chem. Abstr., 114,157224 w, (1991)].

17. H. A. Stefani, C. B. Oliveira, R. B. Almeida, C. M. P. Pereira, R. C. Braga, R. Cella, V. C. Borges, L. Savegnago, and C. W. Nogueira, Eur. J. Med. Chem. 41, 513 (2006). http://dx.doi.org/10.1016/j.ejmech.2006.01.007

18. A. D. Patil, N. V. Kumar, W. C. Kokke, M. F. Bean, A. J. Freyer, C. De Brosse, S. Mai, A. Truneh, D. J. Faulkner, and B. Carte, J. Org. Chem. 60, 1182 (1995). http://dx.doi.org/10.1021/jo00110a021

19. P. Biginelli, Gazz. Chim. Ital. 23, 360 (1893).

20. E. H. Hu, D. R. Sidler, and U.-H. Dolling, J. Org. Chem. 63, 3454 (1998). http://dx.doi.org/10.1021/jo970846u

21. J. Lu and H. Ma, Synlett, no. 1, 63 (2000).

22. B. C. Ranu, A.Hajra, and U. Jana, J. Org. Chem. 65, 6270 (2000). http://dx.doi.org/10.1021/jo000711f

23. K. Ramalinga, P. Vijayalakshmi, and T. N. B. Kaimal, Synlett, no. 6, 863 (2001).

24. J. Lu, Y. Bai, Z. Wang, B.Yang, and H. Ma, Tetrahedron Lett. 41, 9075 (2000). http://dx.doi.org/10.1016/S0040-4039(00)01645-2

25. J. S. Yadav, B. V. S. Reddy, R. Srinivas, C. Venugopal, and T. Ramalingam, Synthesis 1341 (2001). http://dx.doi.org/10.1055/s-2001-15229

26. K. A. Kumar, M. Kasthuraiah, C. S. Reddy, and C. D. Reddy, Tetrahedron Lett. 42, 7873 (2001). http://dx.doi.org/10.1016/S0040-4039(01)01603-3

27. J. S. Yadav, B. V. S. Reddy, K. B. Reddy, K. S. Raj, and A. R. Prasad, J. Chem. Soc., Perkin Trans. 1, 1939 (2001). http://dx.doi.org/10.1039/b102565c

28. Y. Ma, C. Qian, L. Wang, and M. Yang, J. Org. Chem. 65, 3864 (2000). http://dx.doi.org/10.1021/j09919052

29. F. Bigi, S.Carloni, B. Frullanti, R. Maggi, and G. Sartori, Tetrahedron Lett. 40, 3465 (1999). http://dx.doi.org/10.1016/S0040-4039(99)00424-4 
30. J. Peng and Y. Deng, Tetrahedron Lett. 42, 5917 (2001). http://dx.doi.org/10.1016/S00404039(01)01139-X

31. H. Salehi and Q.-X. Guo, Synth. Commun., 34, 171 (2004) http://dx.doi.org/10.1081/SCC$\underline{120027250}$

32. J. S. Yadav, B. V. S. Reddy, J. J. Naidu, and K. Sadashiv, Chem. Lett. 33, 926 (2004). http://dx.doi.org/10.1246/cl.2004.926

33. P. Salehi, M. Dabiri, M. A. Zolfigol, and M. A. B. Fard, Tetrahedron Lett., 44, 2889-2891 (2003). http://dx.doi.org/10.1016/S0040-4039(03)00436-2

34. M. M. Heravi, K. Bakhtiari, and F. Z. Bamoharram, Catal Commun. 7, 373 (2006). http://dx.doi.org/10.1016/j.catcom.2005.12.007

35. S. Johne, Pharmazie 36583 (1981).

36. A. Mannschreck, H. Koller, G. Stuhler, M. A. Davies, and J. Traber, Eur. J. Med. Chem. 19, 381 (1984).

37. Q. Chao, L. Deng, H. Shih, L. M. Leoni, D. Genini, D. A. Carson, and H. B. Cottam, J. Med. Chem. 423860 (1999). http://dx.doi.org/10.1021/jm9805900

38. J. Cizmarik and J. Trupe, Pharmazie 42, 139 (1987).

39. P. P. Kung, M. D. Casper, K. L. Cook, L. Willson-Lingardo, L. M. Risen, T. A. Vickers, and R. Ranken, L.B.Blyn, J.R. Wyatt, P.D. Cook and D.J. Ecker, J. Med. Chem. 42, 4705 (1999). http://dx.doi.org/10.1021/jm9903500

40. J. W. Corbett, S. S. Ko, J. D. Rodgers, L. A. Gearhart, N.A. Magnus, L. T. Bacheler, S. Diamond, S. Jeffrey,R. M. Klabe, B. C. Cordova, S. Garber, K. Logue, G. L.Trainor, P. S. Anderson, and K. Erickson-Viitanen, J. Med.Chem. 43, 2019 (2000). http://dx.doi.org/10.1021/jm990580e

41. R.Y. Yang and A. Kaplan, Tetrahedron Lett. 41, 7005 (2000). http://dx.doi.org/10.1016/S00404039(00)01201-6

42. M. Dabiri, P. Salehi, M. S. Khajavi, and A. A.Mohammadi, Heterocycles 63, 14171421 (2004). http://dx.doi.org/10.3987/COM-04-10042

43. P. Salehi, M. Dabiri, M. A. Zolfigol, and M. Baghbanzadeh, Tetrahedron Lett. 46, 7051 (2005). http://dx.doi.org/10.1016/j.tetlet.2005.08.043

44. M. Dabiri, P. Salehi, A. A. Mohammadi, and M. Baghbanzadeh, Synth. Commun. 35, 279 (2005). http://dx.doi.org/10.1081/SCC-200048462

45. M. Dabiri, P. Salehi, A. A. Mohammadi, M. Baghbanzadeh, and G. Kozehgiry, J. Chem. Res. (S) 570 (2004).

46. J. P. Mayer, G. S. Lewis, M. J. Curtis, and J.W. Zhang, Tetrahedron Lett. 38, 8445 (1997). http://dx.doi.org/10.1016/S0040-4039(97)10276-3

47. S. Makino, N. Suzuki, E. Nakanishi, and T. Tsuji, Synlett 1670 (2000). http://dx.doi.org/10.1055/s-2000-7941

48. M. S. Khajavi, A. A. Mohammadi, and S. S. Sadat Hosseini, Synth Commun. 31, 3647 (2001). http://dx.doi.org/10.1081/SCC-100107014

49. F. R. Alexander, A. Berecibar, R. Wrigglesworth, and T. Besson, Tetrahedron 59, 1413 (2003). http://dx.doi.org/10.1016/S0040-4020(03)00053-X

50. J. S. Yadav and B. V. S. Reddy, Tetrahedron Lett. 43, 1905 (2002). http://dx.doi.org/10.1016/S0040-4039(02)00135-1

51. H. Hazarkhani and B. Karimi, Tetrahedron 59, 4757 (2003). http://dx.doi.org/10.1016/S00404020(03)00696-3

52. P. Lidstrom, J. Tierney, B. Wathey, and J. Westman, Tetrahedron 57, 9225 (2001). http://dx.doi.org/10.1016/S0040-4020(01)00906-1

53. D. Adam, Out of the kitchen, Nature 421, 571 (2003). http://dx.doi.org/10.1038/421571a

54. B. L. Hayes, Microwave Synthesis: Chemistry at the Speed of Light (CEM Publishing: Matthews, NC, 2002).

55. P. Salehi, M. Dabiri, A.R. Khosropour and P. Roozbehniya, J. Iranian Chem. Soc. 3(1), 98 (2006).

56. A. W. Bauer, W. M. M. Kirby, J. C. Sherris and M. Turck, Am. J. Clin. Path. 45, 493 (1966). 
57. R. K. Grover and J. D. Moore, Phytopathology 52, 876 (1962).

58. A. N. Dadhania, V. K. Patel and D. K. Raval, J. Braz. Chem. Soc. 22(3), 511 (2011). http://dx.doi.org/10.1590/S0103-50532011000300014 